\title{
Nível de maturidade e comparação dos PDPs de produtos automotivos
}

\author{
Heitor Luiz Murat de Meirelles Quintella \\ UFF \\ Henrique Martins Rocha \\ FEG-UNESP
}

\begin{abstract}
Resumo
O desenvolvimento de novos produtos, que atendam às necessidades do mercado consumidor, é atividade estratégica para sustentabilidade das organizações. 0 objetivo deste artigo é comparar e avaliar o nível de maturidade desses processos em duas montadoras de veículos instaladas na região Sul Fluminense do País. São discutidas algumas características dos processos, expõe-se o modelo de avaliação, baseado nos critérios do CMMI (Capability Maturity Model Integration], e são apresentados os resultados de pesquisa que investigou o nível de maturidade junto a 47 representantes das empresas estudadas. Como resultado, foi possível identificar lacunas na estruturação dos PDP's, com diversas práticas e ferramentas usadas de forma isolada e não integradas, havendo campo suficiente - no entendimento dos próprios executivos - para serem aprimorados e refinados, o que permitiria torná-los mais completos, abrangentes e potentes para alavancar os resultados de mercado e financeiros das próprias organizações.
\end{abstract}

\section{Palavras-chave}

Desenvolvimento de produtos, modelos de maturidade, qualidade, CMMI.

\section{Automotive product development process: comparing and evaluating the maturity level}

\begin{abstract}
The development of new products, which shall fulfill the customer needs, is a strategic activity for the organizations sustainability. This research has the intention of comparing and evaluating the maturity level of the product development process, at two automotive plants in the Southern region of the State of Rio de Janeiro in Brazil. Some of the processes characteristics are discussed, as well as the evaluation model, which is based on the CMMI (Capability Maturity Model Integration] criteria, and the results of the research, which investigated the level of maturity along with 47 representatives from the studied companies, are hereby presented. This study revealed the existence of gaps in the PDP structure, with several practices and tools being used in a non-integrated way, having according to the executives, room for improvement in order to make them more complete, and powerful enough to leverage the market and the financial organization results.
\end{abstract}

Key words

Product development, maturity models, quality, CMMI. 


\section{INTRODUÇ̃̃̃O}

Foco de atenção na literatura acadêmica e empresarial (SENANAYAKE; LITTLE, 2001), o processo de desenvolvimento de produtos (PDP) é uma atividade estratégica ligada ao atendimento das necessidades do consumidor (RABECHINI JR.; CARVALHO; LAURINDO, 2002; BROWNING; FRICKE; NEGELE, 2006): identificar seus desejos e necessidades e responder com produtos inovadores, bem projetados e executados (MAY-PLUMLEE; LITTLE, 2006; KOUFTEROS; MARCOULIDES, 2006), com características de desempenho, custo e distribuição adequadas (MUNDIM et al., 2002), exige das empresas o uso de PDPs que integrem e alinhem seus recursos, de forma estruturada e única para as necessidades da organização, em que ferramentas e métodos distintos são utilizados (CALANTONE; VICKERY; DROGE, 1995; ROZENFELD; SCALICE; AMARAL, 2005). Neste artigo, estuda-se o PDP utilizado na indústria automobilística, escolhida por sua importância na vida cotidiana e relevância econômica (SILVA, 2001b; ALVAREZ; PROENÇA; ANDEREZ, 2002).

do Sul) e o Fox da Volkswagen (DIAS; SALERNO, 2003; CARVALHO, 2003; CONSONI, 2004).

O presente artigo discute os PDPs das montadoras existentes na região Sul Fluminense: uma empresa voltada ao segmento de automóveis, instalada no município de Porto Real, e outra no segmento de veículos comerciais, instalada no Pólo Industrial do município de Resende. Unidades precursoras de toda uma nova geração de montadoras no Brasil: utilizando conceitos modernos de manufatura integrada e parcerias, a estas se seguiram unidades na Bahia e no sul do País. É feita ainda a avaliação do nível de maturidade dos PDPs, tendo como base os critérios do CMMI (Capability Maturity Model Integration), para o que adotou-se a premissa da existência nas mesmas de métodos estruturados para desenvolvimento de seus produtos.

\section{CONTEXTO E FORMULAC̣̃̃O DA SITUAC̣̃̃O PROBLEMA}

Para Cooper (1994), Calantone, Vickery e Droge (1995) e Shulman (2003), novos produtos são fator primordial nos resultados financeiros e de mercado das organizações. No entanto, segundo Baxter (2003), de cada dez idéias sobre novos produtos, três serão desenvolvidas, 1,3 será lançada no mercado e apenas uma

A história da produção automotiva no Brasil iniciou-se, segundo Alvarez, Proença e Andérez (2002), em 1919, quando a Ford iniciou suas operações de montagem no País, em regime de CKD (complete knock-down), seguida em 1925 pela General Motors. Como a demanda reprimida do pós-guerra impulsionou o crescimento das importações de veículos, afetando a balança de pagamentos, o governo implementou restrições alfandegárias, o que levou ao início da fabricação local, com a instalação em 1953 de Volkswagen, Mercedes e Willys-Overland. Esse processo se ampliou nas décadas seguintes e o Brasil possui hoje unidades de produção de todos os principais fabricantes de automóveis: oito dos dez maiores fabricantes de comerciais leves e cinco dos maiores grupos montadores de caminhões, sendo o país com maior diversidade de marcas de automóveis produzidas no mundo (ALVAREZ; PROENÇA; ANDEREZ, 2002).

Segundo Dias e Salerno (2003), as competências desenvolvidas no período do mercado fechado no Brasil permitiram o desenvolvimento dos primeiros produtos "locais", baseados em plataformas existentes: a Brasília e o Gol. Nos últimos anos, foram desenvolvidos localmente modelos de sucesso de mercado como EcoSport (Ford), Celta e Meriva (GM), Pálio (parcialmente desenvolvido no Brasil pela Fiat), o Polo Sedan (desenvolvido sob a responsabilidade da engenharia brasileira, para os mercados de Brasil, China e África será lucrativa, conforme pesquisa realizada junto a 500 empresas nos anos 90 pelo Design Council da Inglaterra, que mostrou que somente $45 \%$ das empresas conseguiam manter os custos de produção dentro das previsões e que somente $49 \%$ conseguiam lançar seus produtos no tempo programado: em média, os produtos custavam 13\% acima do orçado e eram lançados com seis meses de atraso. Desta forma, de acordo com Silva (2001a, p. 2), para ser fonte de competitividade, o PDP precisa ser eficiente e eficaz, sendo necessária a "utilização de metodologias e técnicas capazes de proporcionar tais atributos".

Krishnan e Ulrich (2001), em extensa pesquisa sobre decisões em PDP, listaram os seguintes fatores críticos de sucesso: preço e posicionamento do produto, identificação e atendimento das necessidades dos clientes, alinhamento organizacional, características do time, otimização do desempenho e criatividade no gerenciamento do projeto. Meybodi (2003) listou ainda qualidade, tempo, competência e custos, enquanto Shenhar et al. (2001) e Quintella e Osório (2002) identificaram como dimensões de sucesso de projetos, o cumprimento de prazos e orçamentos, qualidade do produto e geração de receita. Costa Júnior e Silva (2003) pesquisaram os fatores de fracasso no PDP, listados pelos autores, como mau planejamento, mau gerenciamento, conceito ruim, má execução, mau uso da pesquisa e tecnologia ruim. 
Enquanto Thomke e Hippel (2002) defendem que o envolvimento do cliente no processo é elemento que usualmente leva à redução do tempo - também defendido por Hippel e Katz (2002) -, Witzenburg (2003) destaca o uso de simulações - também proposto por Versprille (2001) e Oxberry (2002) - e de testes de subconjuntos e subsistemas separadamente, de forma a antecipar as respostas do produto final, como ocorrido no Global Vehicle Development Process da GM, em que reduziu-se o tempo entre a aprovação do conceito e o início da produção de 48 meses para 18-24 meses, permitindo adequação mais rápida às necessidades de alteração ou validação de tecnologia e ampliando a confiabilidade do produto e do próprio PDP.

Quanto à confiabilidade no processo de desenvolvimento, Krishnan e Bhattacharya (2002) propuseram o uso de ferramentas estocásticas para selecionar a tecnologia, a qual pode variar de robusta e comprovada a incerta (mas com possibilidade de alavancagem competitiva do produto), enquanto MacCormack, Verganti e Iansiti (2001) desafiaram o paradigma de que as mudanças nos projetos devem ser minimizadas. Segundo os autores, "a incerteza e o ambiente dinâmico representam desafios fundamentais" e propõem investimentos no desenvolvimento da arquitetura, junto ao constante feed-back durante as diversas fases de desenvolvimento e possibilidade constante de flexibilidade no produto, que resultariam em melhores projetos e produtos desenvolvidos.

Baxter (2003) recomenda o estabelecimento de metas claras, concisas, específicas e verificáveis ao longo do PDP e que o processo seja estruturado de forma a "afunilar as decisões", mantendo baixo comprometimento financeiro inicial - devido ao alto risco e incerteza existente -, até que, com a maturação do desenvolvimento e conseqüente diminuição do risco, passe-se a efetuar investimentos de maior porte (protótipos, ferramentas de produção, etc.). Gantewerker e Manoski (2003) expõem que a falha nas avaliações de viabilidade dos projetos "cria uma fundação fraca para o projeto, levando normalmente a interações múltiplas e significativo retrabalho". Os autores defendem a quebra do desenvolvimento em fases - da mesma forma que Ulrich e Eppinger (2000) - para, ao avaliar-se cada fase, "filtrar projetos e eliminar os que não serão bem sucedidos no mercado", num processo de afunilamento.

Infere-se, pois, que processos mais maduros para desenvolvimento de produtos nas organizações aumentem a chance de sucesso e retorno financeiro de seus novos produtos. Para tanto, é necessário um adequado planejamento, execução e controle do PDP, permitindo, de forma eficiente e eficaz, gerar resultados satisfatórios para a organização. Os resultados, se gerados de forma continuada, sem a dependência exclusiva de participação e envolvimento de pessoas específicas, indicaria a maturidade desse processo na organização em questão. Faz-se necessário então identificar o grau de maturidade dos PDP, ou seja, a capacidade de serem definidos, utilizados, gerenciados, repetidos, com resultados previsíveis e que possam ser continuamente melhorados.

\section{EMPRESAS ESTUDADAS}

As informações a seguir foram coletadas em entrevistas exploratórias com representantes da área técnica, nacionalização e desenvolvimento de produtos nas montadoras estudadas, entre 2004 e 2006 (dois representantes de cada empresa).

A montadora do segmento automóveis, inaugurada em fevereiro de 2001, conta com quatro unidades de produção: chaparia, pintura, montagem e motores (esta última inaugurada em março 2002), numa área de $150.000 \mathrm{~m}^{2} \mathrm{e}$ população aproximada de 1.800 pessoas, com capacidade de produzir 134.000 veículos/ano, dentre os três modelos atuais (cerca de 250 unidades diárias), para o mercado local e exportação.

\section{Enecessário um adequado planejamento, execução e controle do PDP}

Seu processo de desenvolvimento inicia-se pelas reuniões de estratégia de produtos, envolvendo representantes de sua sede na Europa, com as áreas locais de Estratégia, Marcas (responsável pelas vendas) e Marketing (responsável pelo estudo mercadológico). À partir das oportunidades identificadas, desdobra-se a estratégia de lançamento de um modelo já existente (no mundo), adequando-o às condições do mercado brasileiro, nas denominadas reuniões de Planning com a Marca, em processo interativo (loopings). Os aspectos técnicos dessas adequações são verificados por meio de "validades veiculares" (testes de Engenharia) e, se aprovadas, devem ser validadas por Marketing e Marcas. Segundo os entrevistados, o PDP utilizado pode ser considerado como "não integrado", consistindo de processos satélite, que são utilizados conforme surjam as necessidades. Com base nas informações e detalhes fornecidos nas entrevistas, foi criado o modelo da Figura 1, como interpretação própria do PDP da montadora em questão.

A fábrica de caminhões e ônibus iniciou suas atividades em 1995 em unidade provisória, passando à unidade definitiva em 1996, numa área de 1 milhão de $\mathrm{m}^{2}$, sendo $110.000 \mathrm{~m}^{2}$ de prédios. Com uma população de mais de 3.000 pessoas e capacidade de produção de 57.600 unidades por ano, produz atualmente cerca de 180 unidades/dia, dentre 23 diferentes modelos (mercado local e exportação). Por conta do alto nível de customização exigido neste mercado, a empresa 
mantém parceria com empresa de menor porte, dedicada ao desenvolvimento de protótipos e modelos especiais de veículos.

Há de se ressaltar que estudo prévio comparativo das mesmas empresas aqui estudadas (QUINTELLA; ROCHA; ALVES, 2005) mostrou que, segundo opinião de seus executivos direta ou indiretamente ligados às atividades de lançamento de novos veículos, o PDP não era um fator crítico de sucesso no segmento automóveis, posição contrária à encontrada nos executivos do segmento caminhões: segundo os mesmos, um "método eficiente de desenvolvimento de produto, utilizando as melhores práticas", seria crítico para o sucesso no lançamento de novos veículos. Os autores inferiram que a diferença de percepções se deve a:

enquanto a empresa do segmento automóveis trabalha na identificação de modelos que atendam às necessidades do mercado brasileiro e utiliza a produção em massa e padronização como forma de alavancagem de seus resultados, restando aos consumidores poucas opções de individualização dos produtos - exceto por cores e opcionais escolhidos -, a outra empresa, voltada ao fornecimento de caminhões e ônibus, trabalha com diversas opções de fornecimento, além das diversas customizações que esse mercado exige, tornando-se muito mais próxima e óbvia a interação existente entre as necessidades dos consumidores e o produto que é desenvolvido e oferecido por esta organização.

Desta forma, havia a expectativa inicial de que o PDP da montadora de caminhões seria mais robusto e maduro que o da montadora de automóveis. No entanto, constatou-se durante as entrevistas exploratórias na primeira que havia também uma percepção de não-integração do processo, ainda que diversas ferramentas tenham sido listadas como parte do PDP, por exemplo: pesquisas quantitativas relacionadas ao desempenho do produto (qualidade, durabilidade, falhas) e satisfação. Mas, no entender dos entrevistados, "sem conseguir resultados satisfatórios para identificar oportunidades de diferenciação da concorrência". Segundo os mesmos, por conta desta situação, utilizam métodos qualitativos (QFD,
Focus Groups e Conselhos Consultivos), com "resultados gratificantes". Assim sendo, o foco do estudo voltou-se para a avaliação do nível de maturidade dos PDPs existentes, e qualquer comparação que pudesse surgir da mesma, ao invés do detalhamento dos processos em si.

\section{MODELOS DE MATURIDADE}

O conceito de níveis de maturidade foi primeiramente proposto por Crosby (1979) no chamado "Aferidor de Maturidade da Gerência de Qualidade", que estabelecia cinco estágios com base nas práticas adotadas. Tais princípios foram adaptados em 1986 pelo SEI - Software Engineering Institute, na Carnegie Mellon University - para criar o CMM (Capability Maturity Model), utilizado para avaliar o processo de desenvolvimento de software (HUMPHREY, 1987).

Outros modelos de maturidade surgiram ao longo do tempo: o modelo de avaliação de capabilidade da Interthink Consulting; o Project Management Maturity Model da PM Solutions (GRANT; PENNYPACKER, 2006) e o de Ibbs e Kwak (2000); o modelo de maturidade de PDP da Pittiglio Rabin Todd \& McGrath (McGRATH, 1998); o IPMA Competency Baseline (ANDERSEN; JESSEN, 2002); o PMMM (Project Management Maturity Model), desenvolvido por Kerzner (2002), o OPM3 (Organizational Project Management Maturity Model), desenvolvido pelo Project Management Institute (PMI, 2003), o modelo de evolução da gestão por processos (GONÇALVES, 2000), o ProjectFRAMEWORK, da ESI International (1999), o Project Management Maturity Assessment Model, da Knapp \& Moore Pty e, mais recentemente, o Modelo de Maturidade para PDP (ROZENFELD; SCALICE; AMARAL, 2005); o Prado-MMGP (Modelo de Maturidade em Gerenciamento de Projetos), desenvolvido por Darci Prado (OLIVEIRA, 2006) e o modelo de níveis de escritórios de projetos, proposto por Rodrigues, Rabechini Jr. e Csillag (2006).

Foram ainda criados outros modelos, baseados no CMM, procurando cobrir outras áreas de interesse (SOTILLE, 2003): o Software Acquisition CMM (SA-CMM), voltado aos processos de seleção, compra e instalação de software de terceiros, o Systems Engineering CMM (SE-CMM), focado

Figura 1: PDP: automóveis.

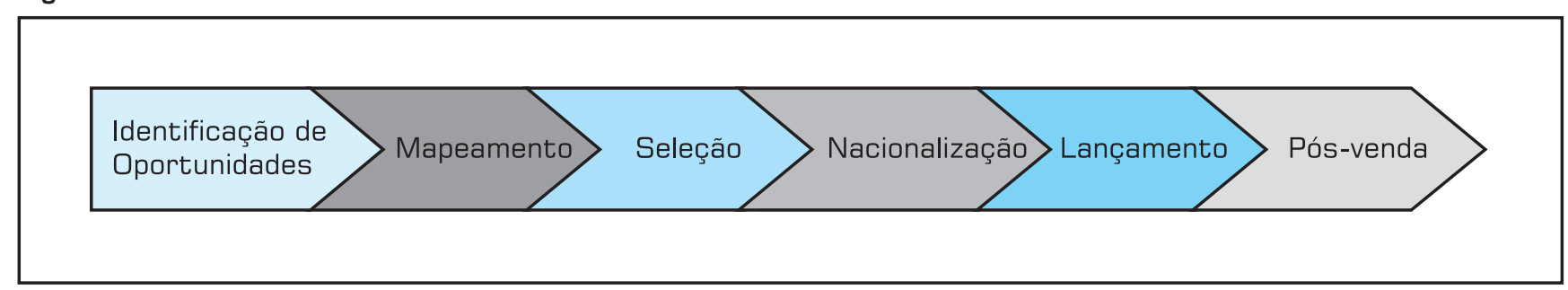

Fonte: elaboração própria. 
nos processos de engenharia de sistemas (hardware, software e quaisquer outros elementos que integram o produto completo), o People CMM (P-CMM), usado nos processos de administração de recursos humanos, e o Integrated Product Development CMM (IPD-CMM), que inclui processos voltados à produção e suporte para integração dos esforços de desenvolvimento de produtos por toda a organização e por todo o ciclo de vida do produto.

Buscando substituir e melhorar estes múltiplos modelos de maturidade que surgiram ao longo dos anos, pelo uso de terminologias, componentes, métodos de avaliação e material de treinamento comuns (SEI, 2004), foi desenvolvido o CMMI - Capability Maturity Model Integration, patrocinado pelo Departamento de Defesa dos Estados Unidos e pelo Comitê de Engenharia de Sistemas da Associação Industrial de Defesa Nacional (NDIA), com a participação, desde 1991, de pessoas de diversas organizações de todo o mundo, as quais utilizavam um ou mais modelos de maturidade.

O CMMI, que "aplica o mesmo conceito de gerenciamento de processos do Six-Sigma e do Total Quality Management" (MILLER; PULGAR-VI-

DAL; FERRIN, 2002, p.1474), com algumas similaridades com a norma ISO 9000:2000 (MUTAFELIJA; STROMBERG, 2003; JAGUARIBE; MARIANO FILHO, 2006), vem desde então tendo crescente utilização no meio empresarial (ZUBROW, 2003; CHRISSIS; KONRAD; SHRUM, 2006), em utilizações não mais limitadas ao desenvolvimento de software (DAVENPORT, 2005). Diversas empresas utilizam-no para estabelecer metas de melhoria de processos internos e padrão para fornecedores (JOKELA; LALLI, 2003), com significativas melhorias de desempenho nas áreas de custo, prazos, qualidade, satisfação de clientes e retorno sobre investimentos (GOLDENSON; GIBSON, 2003). Para a realização da presente pesquisa foi adotada a premissa de que tal modelo é adequado também à mensuração do nível de maturidade do PDP das montadoras estudadas.

\section{Componentes e estrutura do CMMI}

O CMMI contém quatro áreas de conhecimento (disciplinas): Engenharia de Sistemas (CMMI-SE), Engenharia de Software (CMMI-SW), Fonteamento de Fornecedores (CMMI-SS) e Desenvolvimento Integrado de Produto e Processo (CMMI-IPPD). Este último, utilizado na presente pesquisa, derivou-se do IPD-CMM e é uma abordagem sistemática que permite a colaboração das partes envolvidas (stakeholders) por toda a vida do produto, de forma a melhor satisfazer as necessidades, expectativas e requerimentos dos clientes (SEI, 2004). Seus componentes são: níveis de maturidade, áreas de processo, metas gerais e específicas, bem como práticas também genéricas e específicas.
Nível de Maturidade (ML) - estágio evolutivo, com objetivos de processos definidos, que fornece as fundações para melhorias a serem empreendidas no estágio seguinte, guiando o crescimento na capabilidade de processo da organização. As características dos ML são mostradas no Quadro 1.

Área de Processo (PAs) - agrupamento de práticas que, quando executadas coletivamente, satisfazem um grupo de metas necessárias à melhoria na área em questão: cada PA contém Metas Específicas (SGs) e Genéricas (GGs), que devem ser alcançadas, para o atingimento dos níveis de maturidade. Para determinação do ML, é avaliado o atendimento das metas das PA's: uma PA é avaliada como "satisfeita" somente se todas as suas GGs e SGs forem avaliadas como "satisfeitas". O não atendimento a qualquer das metas, faz com que a área de processo como um todo seja avaliada como "não satisfeita" (SEI, 2001b), visto que os processos "não alcançam seus potenciais totais, até que a base adequada seja estabelecida" (SEI, 2001a) e, desta forma, tendem a falhar em condições de estresse.

\section{$\Lambda$ mbas as organizações alcançaram, pelos critérios do CMMI, o ML2 - Gerenciado - para seu PDP}

O Quadro 2 mostra as PAs correspondentes a cada nível de maturidade, bem como as metas genéricas. As SGs, únicas para cada PA, são apresentadas no Apêndice B.

Para o atingimento das metas, o modelo recomenda a utilização de Práticas diversas, específicas e genéricas, mas que não são objeto de verificação nos processos de avaliação do nível de maturidade.

\section{MÉTODOS E TÉCNICAS DA PESQUISA}

Buscando averiguar a existência de um método estruturado para desenvolvimento de produtos nas montadoras estudadas e avaliar o nível de maturidade do mesmo, realizou-se a pesquisa junto a gerentes e corpo técnico de Marketing, Produto, Logística, Suprimentos e Manufatura das mesmas. Consistindo de um conjunto pequeno, mas de importância chave nas atividades relativas ao PDP das montadoras estudadas, este Universo de profissionais detém grande experiência local em lançamento de produtos, além do conhecimento empírico e experimental sobre o tema.

Um questionário com 27 perguntas foi aplicado, buscando-se avaliar a percepção dos respondentes quanto ao atendimento ou satisfação das metas (genéricas e específicas) 
das PAs dos MLs, com base nos conceitos do CMMI, para determinar-se o nível de maturidade da organização. Para as respostas, estabeleceu-se uma escala ordinal (Likert), para avaliar o grau de concordância/discordância das afirmações relacionadas com o objeto pesquisado (MATTAR, 1996): 1 Não/Nunca; 2 - Raramente; 3 - Às vezes; 4 - Freqüentemente; e 5 - Sempre.
Buscou-se reduzir potenciais distorções nas respostas por meio de contato prévio com os respondentes, quando se ressaltou a importância da imparcialidade e do sigilo da pesquisa. Evitou-se exercer qualquer influência sobre os mesmos, de modo a não influenciar e criar viés em suas respostas.

\section{Quadro 1: Características dos ML.}

\begin{tabular}{|c|}
\hline ML 1 - INICIAL \\
\hline $\begin{array}{l}\text { - Práticas de gestão não estabelecidas; } \\
\text { - Postura reativa frente aos compromissos; } \\
\text { - Planejamento ineficiente; } \\
\text { - Ambiente não estável para desenvolvimento de processos. }\end{array}$ \\
\hline ML 2 - GERENCIADO \\
\hline $\begin{array}{l}\text { - Processos e projetos planejados, executados, medidos e controlados; } \\
\text { - Compromissos estabelecidos conforme as necessidades dos stakeholders; } \\
\text { - Requerimentos, produtos e serviços gerenciados; } \\
\text { - Validação do atendimento aos requisitos, padrões e objetivos por revisão dos stakeholders; } \\
\text { - Práticas existentes são mantidas (mesmo em momentos de crise); } \\
\text { - Status dos produtos e serviços são visíveis para a gerência em pontos específicos (milestones). }\end{array}$ \\
\hline ML 3 - DEFINIDO \\
\hline $\begin{array}{l}\text { - Processos padrão caracterizados, documentados, compreendidos, integrados, descritos conforme padrões, } \\
\text { procedimentos, ferramentas e métodos e melhorados ao longo do tempo; } \\
\text { - Gerência estabelece objetivos de processo baseados no conjunto de processos padrão e assegura-se que } \\
\text { esses objetivos são seguidos de forma apropriada; } \\
\text { - Programa de treinamento para garantir conhecimentos e habilidades requeridas para papéis designados. }\end{array}$ \\
\hline ML 4 - QUANTITATIVAMENTE GERENCIADO \\
\hline $\begin{array}{l}\text { - Objetivos quantitativos de qualidade para os produtos e performance de processos são baseados nas } \\
\text { necessidades dos clientes, usuários finais, implementadores de processo e da própria organização como um } \\
\text { todo; } \\
\text { - Produtividade e qualidade medidas nas atividades importantes em todos os projetos, como processo } \\
\text { gerencial (medidas de desempenho]; } \\
\text { - Produtos e processos compreendidos em termos estatísticos, reduzindo a variação no desempenho para } \\
\text { limites quantitativos aceitáveis, e gerenciados ao longo de toda a vida; } \\
\text { - Riscos na introdução de um novo produto conhecidos e gerenciados. }\end{array}$ \\
\hline ML 5 - EM OTIMIZAÇÃO \\
\hline $\begin{array}{l}\text { - Estabelecidos objetivos mensuráveis de melhoria de processos, continuamente revisados para refletir } \\
\text { mudanças nos objetivos de negócio; } \\
\text { - A organização inteira está focada na melhoria contínua da performance de processo, por melhoria } \\
\text { incremental e por inovações tecnológicas; } \\
\text { - Dados sobre a efetividade dos processos usados para realizar análises de custo-benefício das novas } \\
\text { tecnologias e das mudanças propostas; } \\
\text { - Lições aprendidas são disseminadas para outros projetos; } \\
\text { - Há otimização de processos, com a participação e empowerment da força de trabalho, alinhada com os } \\
\text { objetivos e valores da organização e seus negócios. }\end{array}$ \\
\hline
\end{tabular}

Fonte: Elaboração própria. 
Foram respondidos 47 questionários, distribuídos conforme mostrado no Gráfico 1 a seguir. As perguntas e a compilação dos resultados para cada organização (AV - automóveis; e UT - caminhões e ônibus) podem ser observadas no Apêndice A.

Para análise dos dados, estabeleceram-se os seguintes critérios:

1. Utilizou-se a mediana como medida de tendência central, visto ter-se uma escala ordinal para respostas (SIEGEL, 1981; HOEL, 1984; LEVIN, 1987; MATTAR, 1996; COOPER; SCHINDLER, 2003);

2. Adotou-se a premissa que as práticas adotadas pelas organizações para caracterizar o atingimento e satisfação de todas as metas das PAs, deveriam ser utilizadas, se não sempre, ao menos na maioria das vezes (as exceções seriam então entendidas como desvios ou não-conformidades isoladas);

3. Desta forma, o ML da organização seria o maior ML cujas medianas de todas as respostas fossem maiores ou iguais a quatro ("freqüentemente" ou "sempre"). Qualquer resposta com mediana inferior a quatro, dentro de um grupo de perguntas, seria interpretada como a não satisfação das metas para o nível, mesmo que a mediana do grupo fosse quatro ou cinco; e

4. Não atingindo o ML2 nas questões, seria entendido que a organização estaria no nível inicial de maturidade (ML1).

Para avaliar a confiança no ML encontrado, deveria-se testar o grau de confiança na mediana ser maior ou igual a quatro; e também que tal valor não tenha sido atingido no ML imediatamente superior: Triola (1999) propõe o teste de sinais para avaliar a mediana de uma única população, enquanto Hoel (1984) cita só ser possível para variáveis contínuas. Como Siegel (1981) defende que em toda escala ordinal há um "continuum" implícito, mesmo que as diferenças dentro das categorias discretas não sejam percebidas, utilizou-se tal teste na presente pesquisa, conforme processo mostrado na Figura 2.

Quadro 2: PAs e GGs por ML.

\begin{tabular}{|c|c|c|c|c|}
\hline \multicolumn{5}{|c|}{ NÍVEL DE MATURIDADE } \\
\hline 1 & 2 & 3 & 4 & 5 \\
\hline \multicolumn{5}{|c|}{ ÁREAS DE PROCESSO } \\
\hline Nenhuma & $\begin{array}{l}\text { Planejamento do } \\
\text { Projeto; } \\
\text { Monitoramento e } \\
\text { Controle do Projeto; } \\
\text { Gerenciamento } \\
\text { de Acordos com } \\
\text { Fornecedores; } \\
\text { Gerenciamento dos } \\
\text { Requerimentos; } \\
\text { Gerenciamento de } \\
\text { Configuração; } \\
\text { Qualidade } \\
\text { Assegurada de } \\
\text { Processo e Produto; } \\
\text { Medição e Análise. }\end{array}$ & $\begin{array}{l}\text { Todas do ML2; } \\
\text { Foco no Processo Organizacional; } \\
\text { Definição do Processo Organizacional; } \\
\text { Treinamento Organizacional; } \\
\text { Gerenciamento Integrado do Projeto; } \\
\text { Gerenciamento de Riscos; } \\
\text { Integração do Time; } \\
\text { Gerenciamento Integrado de } \\
\text { Fornecedores; } \\
\text { Desenvolvimento dos Requerimentos; } \\
\text { Solução Técnica; } \\
\text { Integração do Produto; } \\
\text { Verificação; } \\
\text { Validação; } \\
\text { Ambiente Organizacional para } \\
\text { Integração; } \\
\text { Análise de Decisão e Resolução. }\end{array}$ & $\begin{array}{l}\text { Todas do ML3; } \\
\text { Performance } \\
\text { de Processo } \\
\text { Organizacional; } \\
\text { Gerenciamento } \\
\text { Quantitativo } \\
\text { do Projeto. }\end{array}$ & $\begin{array}{l}\text { Todas do ML4; } \\
\text { Inovação e } \\
\text { Desdobramento } \\
\text { Organizacional; } \\
\text { Análise Causal } \\
\text { e Resolução. }\end{array}$ \\
\hline \multicolumn{5}{|c|}{ METAS GENÉRIOAS } \\
\hline Nenhuma & $\begin{array}{l}\text { Processo } \\
\text { institucionalizado } \\
\text { como um processo } \\
\text { gerenciado }\end{array}$ & \multicolumn{3}{|c|}{ Processo institucionalizado como um processo definido } \\
\hline
\end{tabular}

Fonte: elaboração própria. 


\section{ANÁLISE DOS RESULTADOS}

Com base no critério da mediana mínima quatro para enquadramento no nível de maturidade - por grupo de perguntas e por pergunta individual -, percebe-se no Apêndice A que ambas as organizações alcançaram, pelos critérios do CMMI, o ML2 - Gerenciado - para seu PDP. Ainda que existissem valores " 4 " em níveis superiores de maturidade, o fato de não atingi-lo em todas as perguntas no ML3 (vide "Menor mediana") caracteriza a não satisfação das metas das PAs deste ML.

Calculou-se então a variabilidade - ou a sensibilidade do valor de tendência central com relação à dispersão (intervalo interquartil $=1$, para ambas as organizações), utilizando-se o coeficiente de variação relativa, conforme a fórmula seguinte:

$$
\mathrm{CV}=\frac{\mathrm{DM}}{\mathrm{Md}} \times 100
$$

O desvio médio por unidade de mediana calculado foi de $16,62 \%$ para "automóveis" e $16,08 \%$ para "caminhões e ônibus". Iniciou-se então o teste de sinais para avaliar a confiança do ML identificado, ou seja, o atingimento da mediana quatro no ML2 e não atingimento no ML3. A compilação das respostas é apresentada na Tabela 1.

Como pode ser observado, na análise do ML2, o número de vezes que o sinal menos freqüente ocorreu correspondeu ao esperado $(62>36$; e $63>29)$, dando-se prosseguimento ao teste, com o cálculo dos valores de "z" (mostrados na mesma tabela). Em seguida, utilizando-se a função "INV. NORMP" do MS Excel, com probabilidade 1\%, encontrouse $\mathrm{z}$ crítico $=-2,326$. Sendo este valor maior que o " $\mathrm{z}$ " de ambas as empresas, conclui-se ao nível de significância $\alpha=0,01$, que estas têm mediana igual ou superior a quatro nas perguntas referentes ao ML2.

No entanto, ao analisar-se as respostas do ML3, constatou-se que o número de ocorrências da mediana superior a quatro era maior que o de inferiores, o que levaria a não rejeição da hipótese nula (ML3 seria alcançado). Tal resultado deveu-se ao fato de que o não atingimento do ML3 se deu não pelo não atingimento da mediana quatro no conjunto de respostas, mas sim pelo não atingimento em algumas respostas individuais (vide Apêndice A, perguntas 12 e 17). Fez-se necessário então analisar separadamente tais respostas, conforme mostrado na Tabela 2.

Os valores de x críticos são mostrados na Tabela 3.

Observa-se que na organização do segmento automóveis, o valor crítico (3) é superior ao número de ocorrências do sinal menos freqüente $(\mathrm{x}=2)$, concluindo-se ao nível de significância $\alpha=0,01$, que a mediana das respostas da pergunta 17 é inferior a quatro, o que corrobora com o não atingimento do ML3. Já na empresa de caminhões e ônibus, não é possível fazer qualquer inferência nesta pergunta, mesmo "relaxando" o nível de significância para 0,05 (x crítico $=5$, contra $\mathrm{x}=6$ ). No entanto, analisada a pergunta 12 , confirma-se o não atingimento do ML3 por esta organização, mas somente ao nível de significância $0,025(\mathrm{x}$ crítico $=\mathrm{x}=3)$.

\section{CONCLUSÕES E RECOMENDAC̄ÕES}

Conclui-se que os PDPs das organizações estudadas, segundo a visão de seus executivos, são equivalentes ao ML2 do CMMI, entendido como sendo um processo "planejado, executado, medido, controlado e com as práticas existentes mantidas, mesmo nos momentos de crise, podendo repetir a experiência para novos projetos". No entanto, os respon-

\section{Gráfico 1: Respondentes.}
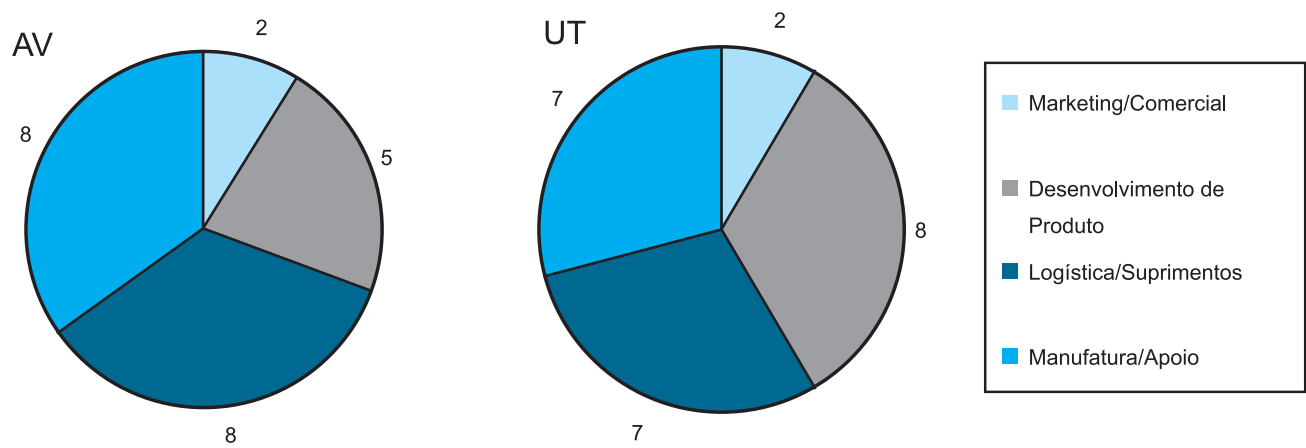

Fonte: Dados da pesquisa. 
Figura 2: Fluxograma para teste de sinais.

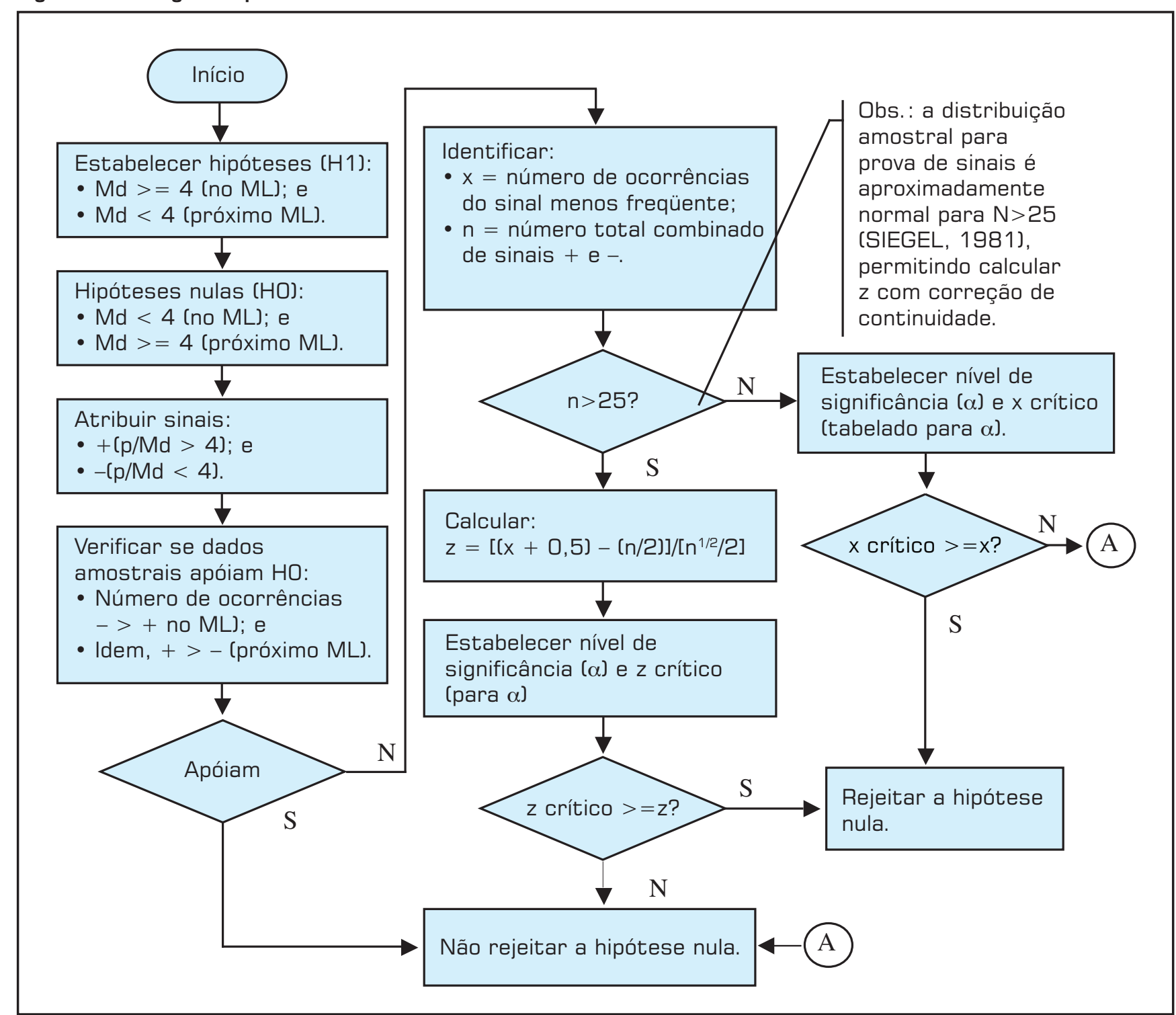

Fonte:Adaptado de Triola (1999).

Tabela 1: Respostas.

\begin{tabular}{|c|c|c|c|c|}
\hline \multirow{2}{*}{ RESPOSTAS } & \multicolumn{2}{|c|}{ AUTOMÓVEIS } & \multicolumn{3}{c|}{ OAMINHOES } \\
\cline { 2 - 5 } & ML2 & ML8 & 29 & 80 \\
\hline 4 & 36 & 73 & 63 & 91 \\
$>4$ & 62 & 90 & $-3,44$ & - \\
\hline
\end{tabular}

Fonte: Dados da pesquisa. 
dentes indicaram que o processo existente não pôde ser caracterizado como integrado e estruturado, nem operar dentro de limites estabelecidos, havendo campo suficiente no entendimento dos próprios executivos - para aprimoramento e refinamento. para torná-los mais completos, abrangentes e potentes para alavancar os resultados de mercado e financeiros da própria organização.

A observação detalhada dos resultados da pesquisa identifica a existência de diferenças significativas de percepção de maturidade no PDP dentre os respondentes. A razão pode ser a dificuldade de visibilidade e entendimento do processo pelas partes envolvidas: apesar das ferramentas e práticas hoje estabelecidas, não existe um processo integrado, que envolva áreas funcionais e de projeto (transversais) das organizações

Tais achados estão alinhados com os insights da pesquisa exploratória, que indicaram a existência de práticas não alinhadas por um processo integrado (processos satélite). $\mathrm{O}$ atingimento do ML3 caracterizaria um PDP definido, ou seja, "caracterizado e compreendido, descrito na forma de padrões, procedimentos, ferramentas e métodos". Recomenda-se pois que ambas as organizações busquem estabelecer PDPs que integrem as diversas práticas e ferramentas existentes (hoje utilizadas de forma isolada), em um modelo único, alinhando recursos e esforços para o desenvolvimento de novos produtos, padronizando ferramentas, métodos, processos e práticas a serem utilizadas por pessoas em funções predefinidas, em momentos específicos. Sugere-se o imediato investimento no desenvolvimento deste PDP, seguido de ações para o desdobramento do mesmo pelas partes envolvidas (internas e externas), por meio de maciço treinamento e acompanhamento da Alta Direção via checkpoints/milestones.

Tabela 2: Respostas individuais.

\begin{tabular}{|c|c|c|c|}
\hline \multirow{3}{*}{ RESPOSTAS } & \multicolumn{3}{|c|}{ PERGUNTA / EMPRESA } \\
\hline & 12 & \multicolumn{2}{|c|}{17} \\
\hline & \multicolumn{2}{|c|}{ GAMINHÕES } & AUTOMÓVEIS \\
\hline$>4[x]$ & 3 & 6 & 2 \\
\hline$<4$ & 12 & 12 & 15 \\
\hline$n$ & 15 & 18 & 17 \\
\hline
\end{tabular}

Fonte: Dados da pesquisa.

Tabela 3: x críticos.

\begin{tabular}{|c|c|c|c|}
\hline \multirow{2}{*}{$n$} & \multicolumn{4}{|c|}{$\alpha$} & 0,025 \\
\cline { 2 - 4 } & 0,01 & 3 & 3 \\
15 & 2 & 4 & 4 \\
17 & 3 & 4 & 5 \\
18 & 3 & 4 & 5 \\
\hline
\end{tabular}

Fonte: Adaptado de Triola (1999, p.365). 


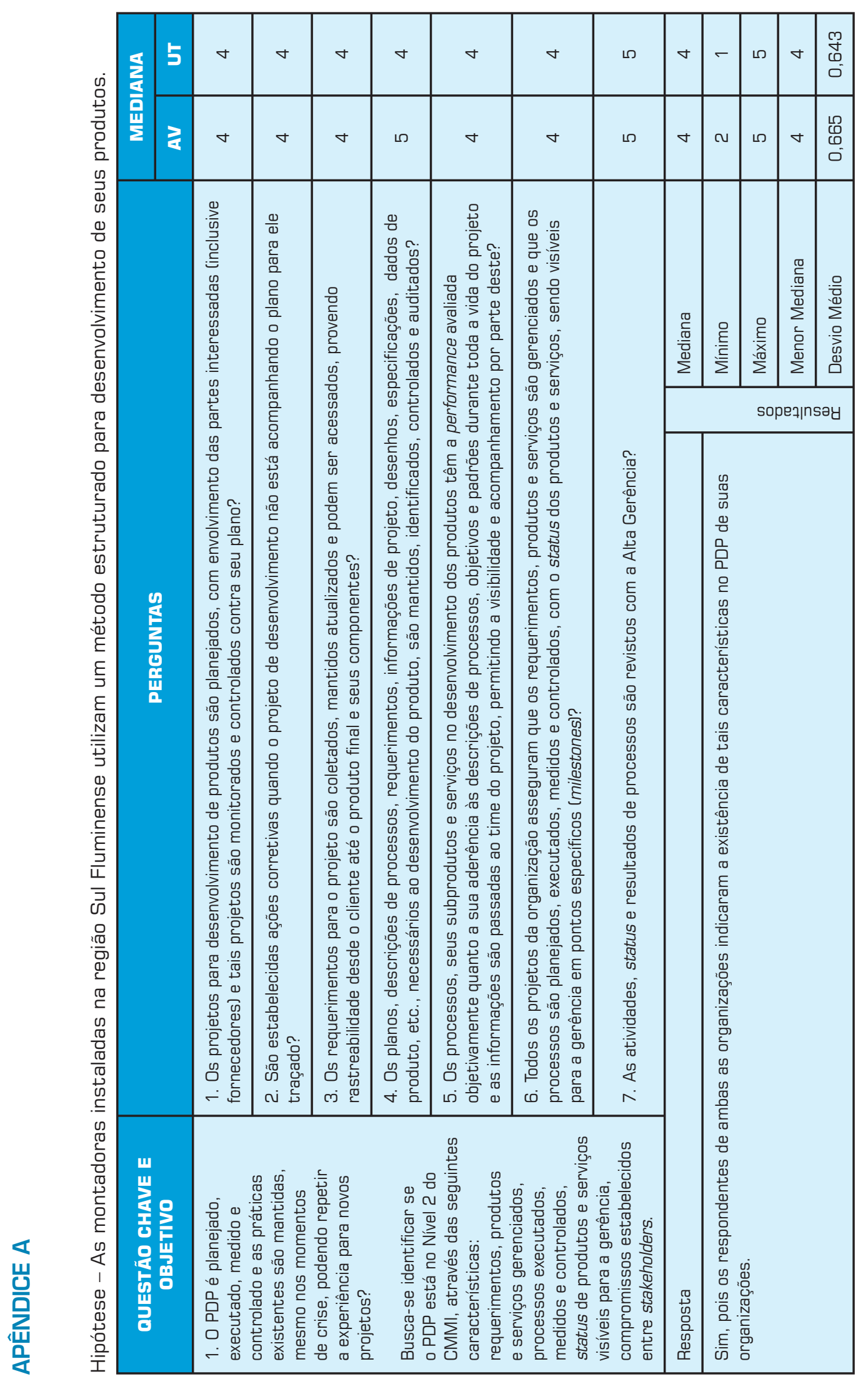




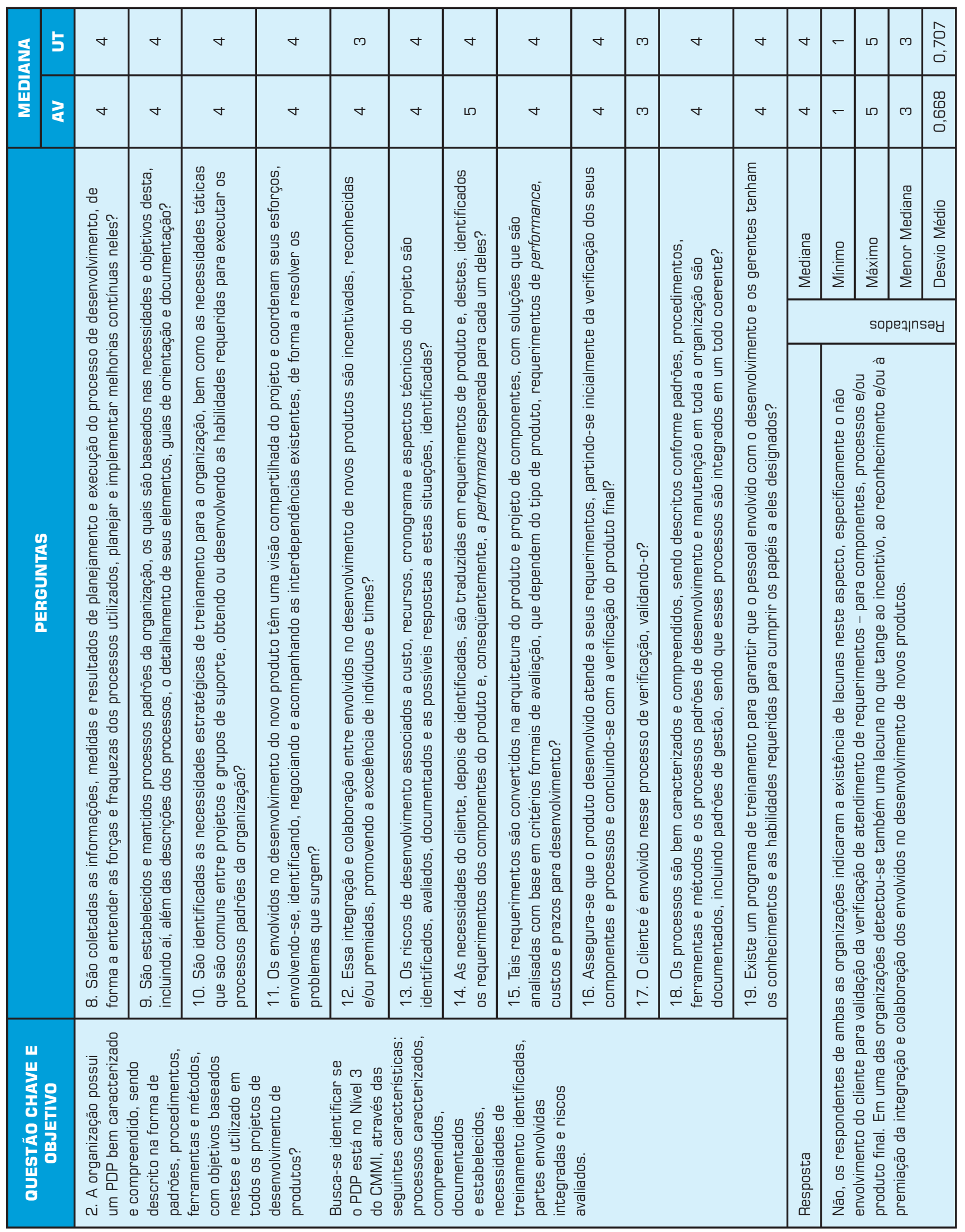




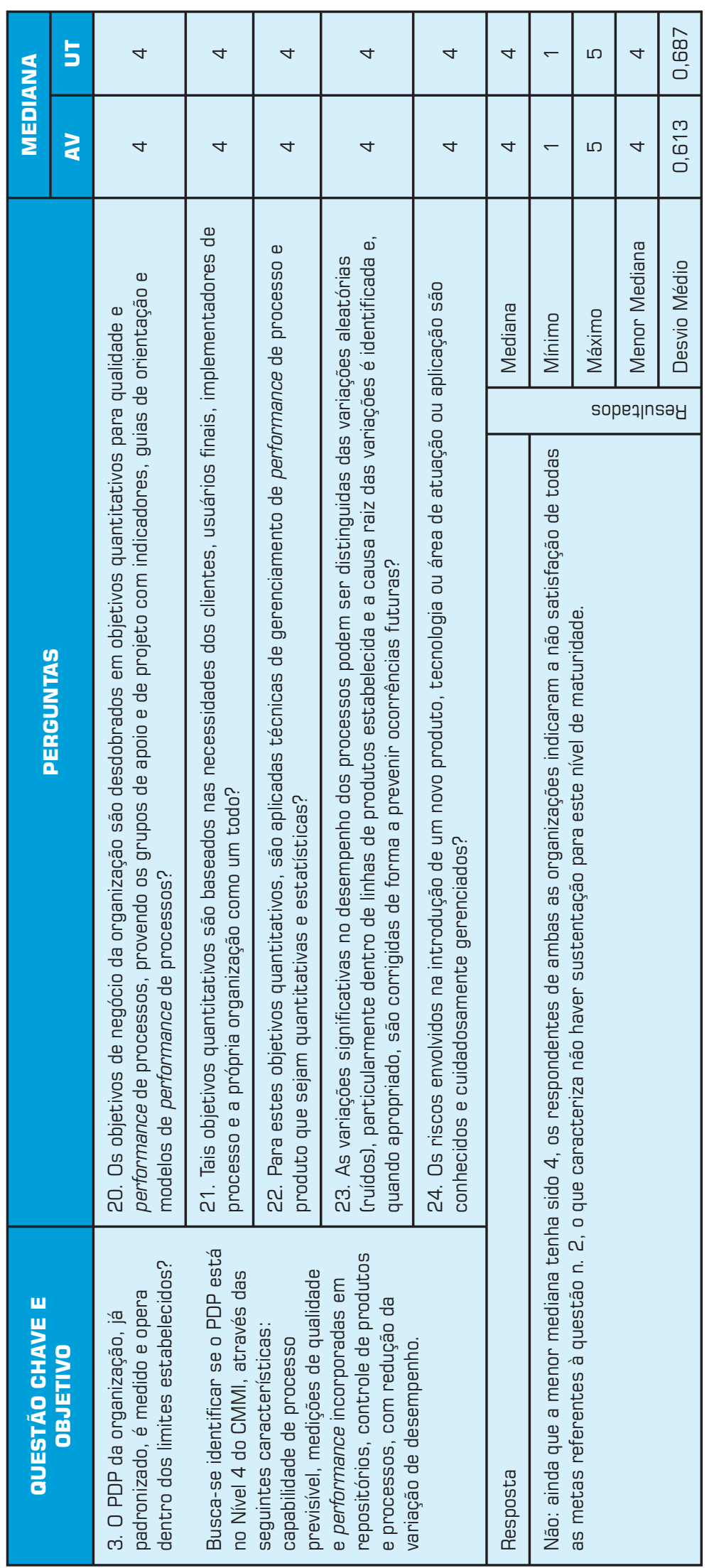




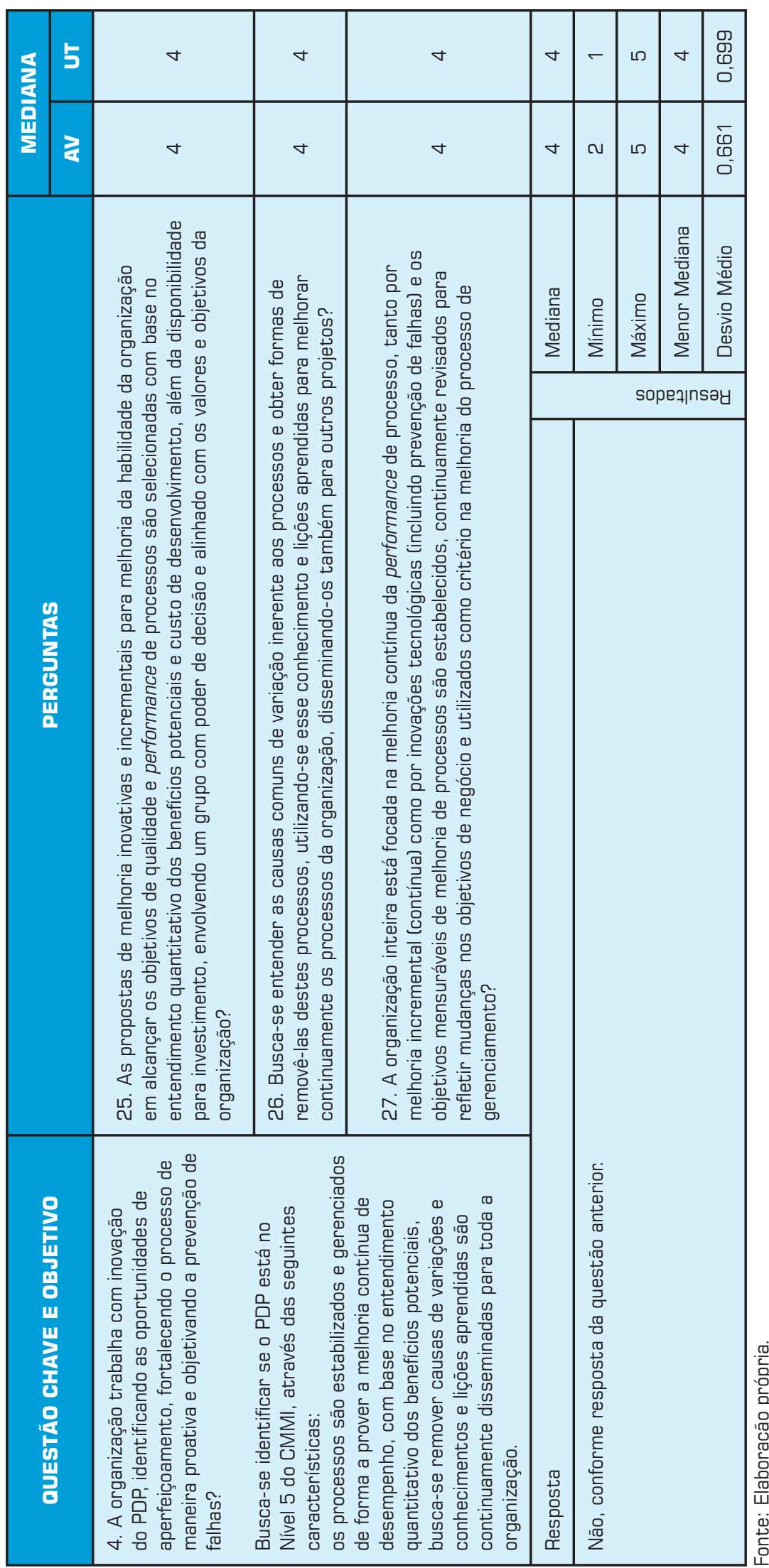


APÊNDICE B — METAS ESPECÍFICAS POR ML.

\begin{tabular}{|c|c|c|}
\hline ML & ÁREAS DE PROCESSO & METAS ESPECIFIGAS \\
\hline \multirow{7}{*}{2} & Planejamento do Projeto & $\begin{array}{l}\text { Estabelecer estimativas; } \\
\text { Desenvolver o plano do projeto; } \\
\text { Obter compromisso com o plano. }\end{array}$ \\
\hline & Monitoramento e Controle do Projeto & $\begin{array}{l}\text { Monitorar projeto contra o plano; } \\
\text { Gerenciar ações corretivas para conclusão. }\end{array}$ \\
\hline & Gerenciamento de Acordos com Fornecedores & $\begin{array}{l}\text { Estabelecer acordos com fornecedores; } \\
\text { Satisfazer acordos com fornecedores. }\end{array}$ \\
\hline & Gerenciamento dos Requerimentos & Gerenciar requerimentos. \\
\hline & Gerenciamento de Configuração & $\begin{array}{l}\text { Estabelecer baselines; } \\
\text { Acompanhar e controlar modificações; } \\
\text { Estabelecer integridade. }\end{array}$ \\
\hline & Qualidade Assegurada de Processo e Produto & $\begin{array}{l}\text { Avaliar objetivamente processos e resultados; } \\
\text { Prover compreensão objetiva. }\end{array}$ \\
\hline & Medição e Análise & $\begin{array}{l}\text { Alinhar atividades de medição e análise; } \\
\text { Prover resultados de medições. }\end{array}$ \\
\hline \multirow{14}{*}{3} & Foco no Processo Organizacional & $\begin{array}{l}\text { Determinar oportunidades de melhoria do processo; } \\
\text { Planejar e implementar atividades de melhoria de processos. }\end{array}$ \\
\hline & Definição do Processo Organizacional & Estabelecer estrutura do processo organizacional. \\
\hline & Treinamento Organizacional & $\begin{array}{l}\text { Estabelecer capabilidade de treinamento organizacional; } \\
\text { Prover treinamento necessário. }\end{array}$ \\
\hline & Gerenciamento Integrado do Projeto & $\begin{array}{l}\text { Utilização do processo definido para o projeto; } \\
\text { Coordenar e colaborar com stakeholders relevantes; } \\
\text { Utilização da visão compartilhada para gerenciamento integrado do projeto }\end{array}$ \\
\hline & Gerenciamento de Riscos & $\begin{array}{l}\text { Preparar para gerenciamento de riscos; } \\
\text { Identificar e analisar riscos; } \\
\text { Mitigar riscos. }\end{array}$ \\
\hline & Integração do Time & $\begin{array}{l}\text { Estabelecer a composição do time; } \\
\text { Gerenciar a operação do time. }\end{array}$ \\
\hline & Gerenciamento Integrado de Fornecedores & $\begin{array}{l}\text { Analisar e selecionar fontes de produtos; } \\
\text { Coordenar trabalhos com fornecedores. }\end{array}$ \\
\hline & Desenvolvimento dos Requerimentos & $\begin{array}{l}\text { Desenvolver requerimentos do cliente; } \\
\text { Desenvolver requerimentos do produto; } \\
\text { Analisar e validar requerimentos. }\end{array}$ \\
\hline & Solução Técnica & $\begin{array}{l}\text { Selecionar soluções de produto e componentes; } \\
\text { Desenvolver o design; } \\
\text { Implementar o design do produto. }\end{array}$ \\
\hline & Integração do Produto & $\begin{array}{l}\text { Preparar para integração do produto; } \\
\text { Assegurar compatibilidade de interface; } \\
\text { Montar componentes e entregar produto. }\end{array}$ \\
\hline & Verificação & $\begin{array}{l}\text { Preparar para verificação; } \\
\text { Executar revisão com pares (peer review); } \\
\text { Verificar resultados de trabalho selecionados. }\end{array}$ \\
\hline & Validação & $\begin{array}{l}\text { Preparar para validação; } \\
\text { Validar produto ou componente do produto. }\end{array}$ \\
\hline & Ambiente Organizacional para Integração & $\begin{array}{l}\text { Prover infra-estrutura para gerenciamento integrado do produto; } \\
\text { Gerenciar as pessoas para a integração. }\end{array}$ \\
\hline & Análise de Decisão e Resolução & Avaliar alternativas. \\
\hline \multirow[b]{2}{*}{4} & Performance de Processo Organizacional & Estabelecer baselines e modelos de performance. \\
\hline & Gerenciamento Quantitativo do Projeto & $\begin{array}{l}\text { Gerenciar quantitativamente o projeto; } \\
\text { Gerenciar estatisticamente a performance de subprocessos }\end{array}$ \\
\hline \multirow{2}{*}{5} & Inovação e Desdobramento Organizacional & $\begin{array}{l}\text { Selecionar melhorias; } \\
\text { Desdobrar melhorias. }\end{array}$ \\
\hline & Análise Causal e Resolução & $\begin{array}{l}\text { Determinar causas dos defeitos; } \\
\text { Endereçar causas dos defeitos. }\end{array}$ \\
\hline
\end{tabular}

Fonte: Elaboração própria. 


\section{Artigo recebido em 02/06/2005 Aprovado para publicação em 12/07/2006}

\section{- Referências}

ALVAREZ, R.; PROENÇA, A.; ANDEREZ, D. Rio Automotivo: Elementos da Realidade e Perspectivas de Desenvolvimento. Rio de Janeiro: SEBRAE, 2002

ANDERSEN, E.; JESSEN, S. Project Maturity in Organisations. Internationa Journal of Project Management, v. 21, p 457-461, 2003.

BAXTER, M. Projeto de Produto: Guia Prático para o Design de Novos Produtos. 2 ed. São Paulo: Edgard Blücher, 2003.

BROWNING, T.; FRICKE, E.; NEGELE, $\mathrm{H}$. Key Concepts in Modeling Product Development Processes. Systems Engineering, v. 9, n. 2, p. 104-128, 2006.

CALANTONE, R.; VICKERY, S.; DROGE, C.. Business Performance and Strategic New Product Development Activities: an Empirical Investigation. Journal of Product Innovation Management, v. 9, n. 2, p. 128 139, Jun. 1995.

CARVALHO, E. Globalização e Estratégias Competitivas na Indústria Automobilística: uma Abordagem a partir das Principais Montadoras Instaladas no Brasil. 2003. $274 \mathrm{f}$. Tese (Doutorado em Economia) Universidade Estadual de Campinas, Campinas, 2003.

CHRISSIS, M.; KONRAD, M.; SHRUM, S CMMI: Guidelines for Process Integration and Product Improvement. 2. ed. Boston: Addison-Wesley, 2006

CONSONI, F. Da Tropicalização ao Projeto de Veículos: um Estudo das Competências em Desenvolvimento de Produtos nas Montadoras de Automóveis no Brasil. 2004, 267 f. Tese (Doutorado em Política Científica e Tecnológica), Universidade Estadual de Campinas, Campinas, 2004.

COOPER, R. Debunking the Myths of New Product Development. Research Technology Management, v. 37, n. 4, p. 45-50, 1994.

COOPER, D.; SCHINDLER, P. Métodos de Pesquisa em Administração, 7. ed. Porto Alegre: Bookman, 2003.

COSTA J. A.; SILVA, C. Os Fatores de Fracasso no Desenvolvimento de Produtos: um Estudo de Caso em uma Pequena Empresa de Alta Tecnologia.
In: IV Congresso Brasileiro de Gestão e Desenvolvimento de Produtos, 2003. Gramado, Anais..

CROSBY, P. Quality is Free. New York: McGraw-Hill, 1979.

DAVENPORT, T. The Coming Commoditization of Process. HBR, v. 83 n. 6, p. 101-108, Jun 2005.

DIAS, A.; SALERNO, M. Produto Mundial, Engenharia Brasileira: Integração de Subsidiárias no Desenvolvimento de Produtos Globais na Indústria Automobilística. Boletim Técnico da Escola Politécnica da USP, São Paulo, n. 169, 2003.

ESI International - Education Services Institute. Project Framework. Washington, DC: George Washington University, 1999.

GANTEWERKER, S.; MANOSKI, P. The Library - Not the Lab: Why it's Important to Do Your Homework Before Hands-on Product Development Work Begins. Food Processing, v. 64, i. 9, p. 40-43, Dec. 2003.

GOLDENSON, D.; GIBSON, D. Demonstrating the Impact and Benefit of CMMI: an Update and Preliminary Results. Special Report CMU/SEI-2003SR-009. SEI, Carnegie Mellon University, 2003.

GONCALVES, J. Processo, que Processo? $R A E$, v. 40 , n. 4, p. 8-19, Out.-Dez. 2000 .

GRANT; K.; PENNYPACKER, J. Project Management Maturity: Assessment of Project Management Capabilities Among and Between Selected Industries. IEEE Transactions on Engineering Management, v. 53, n. 1, p. 59-68, Feb. 2006.

HIPPEL, E.; KATZ, R. Shifting Innovation to Users via Toolkits. Management Science, v. 48 , i. 7 , p. 821-834, Jul. 2002.

HOEL, P. Estatística Matemática, 4. ed. Rio de Janeiro: Guanabara Dois, 1984.

HUMPHREY, W. Characterizing the Software Process: a Maturity Framework. CMU/SEI-87-TR-11, ADA182895, SEI, Carnegie Mellon University, Jun. 1987.
IBBS, W.; KWAK, Y.-H. Assessing Project Management Maturity. Project Management Journal, v. 31, p. 32-43, Mar. 2000.

JAGUARIBE, M.; MARIANO FILHO, Luiz. Determinação da Maturidade de Processos em Empresas Certificadas pela NBR ISO 9001:2000 como um Indicador da Gestão por Processos. In: III Simpósio de Excelência em Gestão e Tecnologia - SEGeT, 2000, Resende, AEDB. Anais..

JOKELA, T.; LALLI, T. Usability and CMMI Does a Higher Maturity Level in Product Development Mean Better Usability? In: Computer-Human Interaction, Ft. Lauderdale, Apr. 2003

KOUFTEROS, X.; MARCOULIDES, G Product Development Practices and Performence: an Structural Equation Modeling-Based Multi-Group Analysis. International Journal of Production Economics, v.103, i.1, p.286-307, Sep.2006.

KRISHNAN, V.; BHATTACHARYA, S. Technology Selection and Commitment in New Product Development: The Role of Uncertainty and Design Flexibility. Management Science, v. 48 , i. 3, p. 313 349, Mar. 2002

KRISHNAN, V.; ULRICH, K. Product Development Decisions: a Review of Literature. Management Science, v. 47, n. 1, p. 1-21, Jan. 2001.

LEVIN, Jack. Estatística Aplicada a Ciências Humanas, 2. ed. São Paulo: Habras, 1987.

MACCORMACK, A.; VERGANTI, R. IANSITI, M. Developing Products on "Internet Time": The Anatomy of a Flexible Development Process. Management Science, v. 47, i. 1, p. 133152, Jan. 2001

MATTAR, F. Pesquisa de Marketing. 2 volumes. São Paulo: Atlas, 1996.

MAY-PLUMLEE, T.; LITTLE, T. Proactive Product Development Integrating Consumer Requirements. International Journal of Clothing Science and Technology, v. 18, n. 1 , p. $53-66,2006$
McGRATH, M. Reviewing up the Product Development. Electronic Business Magazine, p. 36, Jan. 1998.

MEYBODI, M. Using Principles of Just-in-Time to Improve New Product Development Process. In: Advances in Competitiveness Research, v. 11, i. 1, p. 116-140, 2003.

MILLER, M.; PULGAR-VIDAL, F.; FERRIN D. Achieving Higher Level of CMMI Maturity Using Simulations. In: INFORMS Proceedings 2002, Winter Simulation Conference, San Diego, 2002.

MUNDIM, A. et al. Aplicando o Cenário de Desenvolvimento de Produtos em um Caso Prático de Capacitação Profissional. Gestão \& Produção, v. 9, n. 1, p. 1-16, abr. 2002.

MUTAFELIJA, B ; STROMBERG, $\mathrm{H}$ Exploring CMMI - ISO 9001:2000. Synergy when Developing a Process Improvement Strategy. In: SEPG 2003 Conference. BearingPoint \& Hughes Network Systems, Boston, p. 7-43, 2003.

OLIVEIRA, W. Modelos de Maturidade: Visão Geral. MundoPM, v. 1, n. 6, p. 6-11, Dez.Jan. 2006.

OXBERRY, E. Rapid Fire: With ever Advancing Rapid Prototyping and Production Technologies, the Development of New Materials is as Important as that of the Management Technology Itself. Plastics Engineering, v. 243, i. 12 , p. 28 , Dec. 2002

PMI - PROJECT MANAGEMENT INSTITUTE. Organizational Project Management Maturity Model. PMI, 2003.

QUINTELLA, H.; OSORIO, R. CMM Qualidade de Produtos de Software na DATAPREV. Relatórios de Pesquisa em Engenharia de Produção, UFF, v.1, n.14 p.1-5, 2002. Disponível em <http:// www.producao.uff.br/rpep/RelPesq/relpesq014.htm>. Acesso em 23 out. 2006

QUINTELLA, H.; ROCHA, H.; ALVES, M. Projeto de Veículos Automotivos: Fatores Críticos de Sucesso. Produção, v. 15, n. 3 , p. 334-346, 2005. 
RABECHINI JR., R.; CARVALHO, M.; LAURINDO, F. Fatores Críticos para Implementação de Gerenciamento por Projetos: o caso de uma Organização de Pesquisa. Produção, v. 12, n. 2, p. 28-41, 2002.

RODRIGUES, I.; RABECHINI JR. R.; CSILLAG, M. Os Escritórios de Projetos como Indutores de Maturidade em Gestão de Projetos. RAUSP, v. 41, n. 3, p. 273-287, jul.-ago.-set. 2006.

ROZENFELD, H.; SCALICE, R.; AMARAL, D. Proposta de um Método de Maturidade para o Processo de Desenvolvimento de Produtos. In: V Congresso Brasileiro de Gestão de Desenvolvimento de Produto, 2005, Curitiba. Anais...

SEI. Capability Maturity Model Integration for Systems Engineering, Software Engineering, and Integrated Product and Process Development (CMMI-SE/ SW/IPPD), v.1.1. SEI, Carnegie Mellon, 2001a.
Standard CMMI Appraisal Method for Process Improvement (SCAMPI), version 1.1. Method Definition Document (CMMI-SEI-2001-HB-001). SEI, Carnegie Mellon, 2001b.

CMMI General Information. SEI Carnegie Mellon, 2004. Disponível em http://www.sei.cmu.edu/cmmi/general/general.html. Acesso em: 10 ago. 2004.

SENANAYAKE, M.; LITTLE, T. Measures for New Product Development. Journal of Textil and Apparel, Technology and Management, v. 1, n. 3, p. 1-14, May 2001.

SHENHAR et al. Project Success: A Multidimensional Strategy Concept. In: Long Range Planning, n. 34, p. 699-725, 2001.

SHULMAN, R. Recovery and The New Product Paradox. Brandweek, v. 44, i. 25 p. 20, Jun. 2003.
SIEGEL, S. Estatística Não-Paramétrica para as Ciências do Comportamento. São Paulo: McGraw-Hill, 1981.

SILVA, C. Método para Avaliação do Desempenho do Processo de Desenvolvimento de Produtos. 2001, 187 f. Tese (Doutorado em Engenharia de Produção), Universidade Federal de Santa Catarina, Florianópolis, 2001a.

SILVA, C. Competitividade e Estratégia Empresarial: um Estudo de Caso na Indústria Automobilística Brasileira na Década de 1990. Rev. FAE, Curitiba, v. 4, n. 1, p. 37-48, Jan.-abr. 2001b.

SOTILLE, M. PMBOK \& CMM + CMMI. Disponível em < http://www.pmtech.com. br>. Acesso em: fev. 2004.

TOMKE, S.; HIPPEL, E. Customers as Innovators: a New Way to Create Value. $H B R$, v. 80, i. 4, p. 74-81, 2002.

TRIOLA, M. Introdução à Estatística, 7. ed. Rio de Janeiro: LTC, 1999.
ULRICH, K.; EPPINGER, S. Product Design and Development, 2. ed, New York McGraw-Hill, 2000.

VERSPRILLE, K. Elevating CAE into the Design Process. Computer-Aided Engineering, v. 20, i. 9, p. 52, Sep. 2001.

WILSON, C.; KENNEDY, M.; TRAMMELL, C. Superior Product Development: Managing the Process for Innovative Products. Cambridge: Blackwell, 1996.

WITZENBURG, G. Vehicle Development View from the Trenches: A Look at the Tricks and Tools Automakers use to Squeeze Time and Cost Out of New Product Development. Automotive Industries, v. 183 , i. 3 , p. 40-43, Mar 2003.

ZUBROW, D. Current Trends in the Adoption of the CMMI Product Suite. In: $27^{\text {th }}$ Annua International Computer Software and Applications Conference, 2003.

\section{- Agradecimentos}

Os autores agradecem os comentários e críticas dos revisores, que contribuíram para a melhoria do artigo. Agradecemos também ao Prof. Dr. Maurício Delamaro (FEG-UNESP), pela revisão final do artigo.

\section{- Sobre os autores}

Heitor Luiz Murat de Meirelles Quintella, D.sc.

Professor da Universidade Federal Fluminense, Centro Tecnológico, Escola de Engenharia.

End.: Rua Passo da Pátria, 156 - Praia Vermelha - 24210-240 - Niterói - RJ - Brasil

Tel.: (21) 2239-0405 Ramal: 5451 Fax: (21) 2629-5451

E-mail: hquintel@unisys.com.br

\section{Henrique Martins Rocha, M.sc.}

Professor da Associação Educacional Dom Bosco, Doutorando da FEG-UNESP.

End.: Estrada Resende Riachuelo, 2535 - Campo de Aviação - 27501970 - Resende - RJ - Brasil

Tel.: (24) 3355-6000 Fax: (24) 3354-0110

E-mail: hmartins@aedb.br 